\title{
Antitumor effect of adenoviral vector prime protein boost immunity targeting the MUC1 VNTRs
}

\author{
YUQIAN WANG ${ }^{1,2}$, CHENLU LIU $^{2}$, QIU XIA ${ }^{2}$, PENG WANG $^{2}$, BO LI $^{2}$, ZHENZHEN LU $^{2}$, JIAXI SUN $^{3}$, \\ HUI $\mathrm{WU}^{2}$, BIN YU ${ }^{2}$, JIAXIN WU ${ }^{2}$, XIANGHUI YU ${ }^{2}$, WEI KONG ${ }^{2}$, HAIHONG ZHANG ${ }^{2}$ and XIANLING CONG ${ }^{1}$ \\ ${ }^{1}$ Science Research Center, China-Japan Union Hospital, Jilin University; ${ }^{2}$ National Engineering Laboratory of AIDS Vaccine, \\ College of Life Science, Jilin University; ${ }^{3}$ High School affiliated with Jilin University, Changchun 130012, P.R. China
}

Received October 17, 2013; Accepted December 9, 2013

DOI: 10.3892/or.2013.2950

\begin{abstract}
Mucin 1 (MUC1) is a tumor-associated antigen that is overexpressed in several adenocarcinomas. However, clinical trials with MUC1 showed that MUC1 is a relatively poor immunogen in humans. In view of the low immunogenicity of this protein vaccine, we designed a method based on an immunoadjuvant and immunization strategy to enhance the cellular immune response to this protein vaccine. DDA/MPL has been evaluated as an adjuvant to induce strong immunity for the tuberculosis vaccine. However, its adjuvant role combined with the vaccine targeting MUC1 in malignant carcinomas has not previously been reported. Our previous study showed that adenovirus prime protein boost vaccination could significantly enhance the cellular immunity and antitumor efficacy. In our study, we used MUC1 VNTRs as the target of cancer vaccine and DDA/MPL as the adjuvant to enhancing the cellular immunity of recombinant MUC1 protein vaccine, and an AD-9M adenoviral vector prime-recombinant protein and DDA/MPL boost (designated MUC-1 VPP vaccine) strategy was studied to enhance the antitumor efficacy. The results demonstrated that antigen-specific IFN- $\gamma$-secreting T cells were increased by 2 -fold, and cytotoxic T lymphocytes (CTLs) were induced effectively when the protein vaccine was combined with the DDA/MPL adjuvant. Moreover, the vaccination induced nearly $60 \%$ inhibition of the growth of B16 melanoma in mice and prolonged the survival of tumor-bearing mice. The inhibition was correlated with the specific immune responses induced by the MUC1 VPP vaccine. The data suggested that DDA/ MPL-adjuvant MUC-1 VPP vaccine may be developed into
\end{abstract}

Correspondence to: Dr Xianling Cong, Science Research Center, China-Japan Union Hospital, Jilin University, Changchun 130012, P.R. China

E-mail: congx1888@hotmail.com

Dr Haihong Zhang, National Engineering Laboratory of AIDS Vaccine, College of Life Science, Jilin University, Changchun 130012, P.R. China

E-mail: zhanghh@jlu.edu.cn

Key words: MUC1 VNTRs, DDA/MPL, vector prime protein boost, melanoma effective tumor vaccines for melanomas and possibly for other tumors expressing MUC1 protein.

\section{Introduction}

Mucin 1 (MUC1) is a classic target for cancer immunotherapy and is under research in many forms in both preclinical and clinical trials $(1,2)$. MUC1 is a transmembrane glycoprotein normally expressed on the apical surface of ductal epithelia. A variable number (20-125) of tandem repeats (VNTRs) of a 20 amino acid sequence was contained in its extracellular domain. On the apical surface of the normal ductal epithelia, VNTRs are heavily glycosylated at threonine and serine residues, up to $70 \%$ carbohydrate by weight, while malignant cells contain underglycosylated VNTR domains, and are overexpressed in $90 \%$ of all adenocarcinomas including breast, lung, pancreas, prostate, stomach, colon and ovary (3). Due to such altered glycosylation, new epitopes appear on the cell surface that are absent in normal tissues. These VNTR-derived epitopes are attractive targets in immunotherapy. Peptide epitopes, containing the PDTR fragment from VNTR of MUC1 have been found to be immunodominant in T-cell and B-cell responses (4). A novel Bacillus Calmette-Guérin-based breast cancer vaccine that coexpresses multiple tandem repeats of MUC1 and CD80 was reported to break the immune tolerance and inhibits MUC1-positive breast cancer growth (5). Mukherjee et al showed that a novel MUC1-based vaccine in combination with a cyclooxygenase-2 inhibitor (celecoxib), and low-dose chemotherapy (gemcitabine) was effective in preventing the progression of preneoplastic intraepithelial lesions to invasive pancreatic ductal adenocarcinomas (6).

Research on protein-based vaccines against cancer has been carried out for many years. However, it is broadly criticized due to the fact that protein vaccines generally induce relatively stronger levels of humoral immunity than cellular immunity, while the latter is considered essential for tumor immunotherapy. Therefore, effective immunoadjuvant and vaccine strategies are required for successful induction of cellular immune responses against the protein vaccine.

Currently, there are many forms of MUC1 vaccine adjuvants which have already been used in clinical trials, such as BCG, QS-21, KLH and SB-AS217. Among them, MPL (monophosphoryl lipid A) activates Toll-like receptor 4 (TLR4) 
and triggers the Trif-dependent pathway (7). Moreover, DDA (dimethyldioctadecylam monium bromide) is a cationic liposome that is able to enhance antigen uptake and presentation (8). MPL formulated with cationic DDA liposomes (DDA/MPL) which was used in the tuberculosis vaccine has been reported to enhance antigen uptake (9), antigen presentation to T cells and stimulate DCs through Toll-like receptors (TLRs) (10). In our previous study, DDA/MPL effectively enhanced the immunity of tumor vaccine targeting survivin (11). However, little was known about its function as the adjuvant for the cancer vaccine targeting MUC1 VNTR. In our study, we used DDA/MPL as the adjuvant for MUC1 protein vaccine. We investigated the immunity enhancement of this adjuvant, both the humoral and cellular aspects.

Our previous experiments also showed that heterologous adenoviral vector prime-recombinant protein and DDA/MPL boost immunization strategy (termed VPP immune strategy) presented better antitumor effects than the homologous adjuvant protein prime-boost immunization (12), which is in accordance with other reports (13-15). Thus, adenoviral vector prime-recombinant protein and DDA/MPL boost was conducted for tumor-suppressive experiments in the present study.

The purpose of our study was to evaluate whether the $9 \mathrm{M}$ protein vaccine induces cellular immune responses and antitumor effects through the optimization of immune adjuvant and immunization strategies. DDA/MPL was used as immunoadjuvant to enhance the immune responses. Adenoviral vector prime-DDA/MPL adjuvant protein boost immune strategy (VPP) was conducted in a murine melanoma model, and the tumor inhibitory effects and mechanisms were studied.

\section{Materials and methods}

Mice, cell lines andantibody. FemaleC57BL/6 mice(6-8 weeks) were purchased from Beijing Huafukang Biology Technology Co. Ltd. Mice were maintained in microisolator cages under pathogen-free conditions, and animal care conformed to the Guide for the Care and Use of Laboratory Animals (National Research Council). Murine C2C12 myoblast cells and B16 melanoma cells were preserved by the National Engineering Laboratory of AIDS Vaccine, Jilin University. MUC1 antibody was purchased from BD Biosciences (550486).

Construction, expression and purification of recombinant protein. The cDNA fragment containing nine identical peptide tandem repeats (sequence HGVTSAPDTRPAPGSTAPPA) was synthesized by Generay Corp. and inserted into pET-26b (Novagen) using the Bam HI and HindIII (Takara) site. Expression of the recombinant MUC1 VNTR protein (9M) was confirmed by immunoblotting using a mouse $\mathrm{mAb}$ against MUC1 VNTR (BD Pharmingen). The recombinant His-tagged protein was purified using a HiTrap chelating HP column (Invitrogen).

Construction of recombinant adenovirus vector expressing MUCl VNTRs. The recombinant adenovirus (rAd) vector expressing MUC1 VNTRs was obtained using the AdMax ${ }^{\mathrm{TM}}$ Adenovirus Vector Creation System (Microbix Biosystems). The 9M fragment with EcoRI/SalI sites was subcloned into the EcoRI/SalI sites of the pDC316 shuttle vector. rAd was produced by homologous recombination between pBHGloxDE1,3Cre, and pDC316-9M at the loxP or frt position when co-transfected into HEK293 cells. Plaque formation observed $\sim 10$ days post-transfection indicated successful generation of the rAd. After verification by PCR or western blot analysis, the rAd containing 9M (AD-9M) was amplified and purified from cell lysates twice in $\mathrm{CsCl}$ density gradients, as previously described $\mathrm{Yu}$ et al (15). Viral products were desalted and stored at $-80^{\circ} \mathrm{C}$ in PBS containing $10 \%$ glycerol $(\mathrm{v} / \mathrm{v})$. The titer of the viral stock was determined using the Reed and Muench method and expressed as 50\% tissue culture infectious doses (TCID50).

qRT-PCR. RNA was extracted by the guanidine isothiocyanate and chloroform method (Invitrogen) from the $\mathrm{C} 2 \mathrm{C} 12$ (a cell line of mice myoblast), B16 cells and implanted B16 tumors. All RNA samples were treated with DNase I (Promega). For all samples, $1 \mu \mathrm{g}$ of total RNA was used to synthesize first-strand cDNA with reverse transcriptase (Invitrogen) and random primers. The cDNA synthesis was performed at $37^{\circ} \mathrm{C}$ for $60 \mathrm{~min}$ after heat inactivation at $95^{\circ} \mathrm{C}$ for $10 \mathrm{~min}$. PCR was performed using 1X SYBR Green PCR Master mix (Transgen) on a realtime PCR system (CFX96; Bio-Rad). MUC1 expression was confirmed by quantitative real-time reverse-transcriptase (RT) PCR (qRT-PCR) using primers 5'-GTGCCCCCTAGCAG TACCG-3' and 5'-GACGTGCCCCTACAAGTTGG-3'. Cycling conditions were: $2 \mathrm{~min}$ at $50^{\circ} \mathrm{C}, 10 \mathrm{~min}$ at $95^{\circ} \mathrm{C}$, and 40 cycles of $15 \mathrm{sec}$ at $95^{\circ} \mathrm{C}$, plus $1 \mathrm{~min}$ at $60^{\circ} \mathrm{C}$. Commercial software (CFX Manager) was used to calculate relative MUC1 expression normalized to the $\beta$-actin endogenous control (primers 5'-CGTGGACATCCGCAAAGACC-3' and 5'-GGACTCGT CATACTCCTGCTTGC-3') for all genes studied using the $2^{-\Delta \Delta \mathrm{Ct}}$ method.

Immunization schedule. $9 \mathrm{M}$ recombinant protein $(50 \mu \mathrm{g})$, $250 \mu \mathrm{g}$ DDA (Arcos Organics) plus $25 \mu \mathrm{g}$ MPL (Avanti Polar Lipids) were injected separately or as a mixture subcutaneously in C57BL/6 mice (5 mice per group) with two week intervals. The mice were vaccinated three times. Two weeks after the final immunization, all mice were sacrificed to detect humoral and cellular immune responses.

Humoral responses. Antigen-specific antibodies in mice sera were estimated by standard enzyme-linked immunosorbent assay (ELISA) when the mice were sacrificed two weeks after the final immunization. The ELISA plates were coated with 9M protein at $0.25 \mu \mathrm{g} / \mathrm{well}$. Sera diluted at 1:100 and horseradish peroxidase (HRP)-conjugated secondary antibody diluted at 1:10,000 (goat anti-mouse IgG, Jackson) were used, and the enzyme reaction was developed with 3,3',5,5'-tetramethylbenzidine (TMB) for 10-15 min and stopped with $2 \mathrm{M} \mathrm{H}_{2} \mathrm{SO}_{4}$. Optical density (OD) was determined with a microplate reader at a wavelength of $450 \mathrm{~nm}$.

IFN- $\gamma$ ELISPOT assay. Releasing of IFN- $\gamma$ from antigenspecific effecter T cells was assessed with an ELISPOT kit (BD Biosciences) according to the manufacturer's instructions. The reaction was terminated upon the appearance of dark purple spots, which were quantitated using the AlphaImager System. 


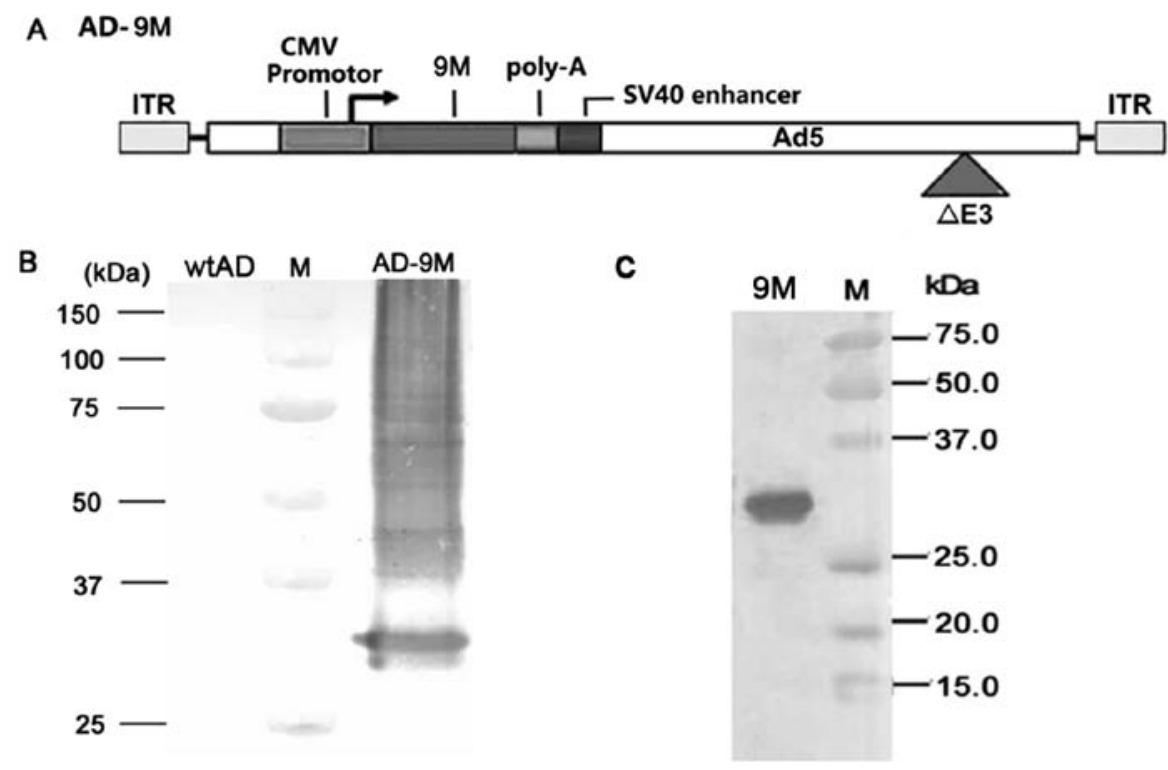

Figure 1. Construction and identification of recombinant adenovirus. (A) AdMax ${ }^{\mathrm{TM}}$ was used for generation of recombinant adenovirus expressing 9M. (B) HEK293 cells were infected with wild-type adenovirus (wtAD) or AD-9M at an MOI 50, incubated for $48 \mathrm{~h}$, and then analyzed by western blotting to detect $9 \mathrm{M}$ expression with the mouse monoclonal anti-MUC1 antibody as the primary antibody.

In vitro cytotoxicity assay. In vitro cytotoxicity was determined as previously reported (16). Briefly, B16 cells used as target cells were pulsed at a concentration of $1 \times 10^{6} \mathrm{cells} / \mathrm{ml}$ with or without $5 \mu \mathrm{g} / \mathrm{ml}$ of the MHC-I H-2Db restricted MUC1 peptide (SAPDTRPAP) in RPMI-1640 medium containing 10\% fetal bovine serum (FBS) for $2 \mathrm{~h}$ at $37^{\circ} \mathrm{C}$. Peptide-loaded B16 cells were then labeled with 5, 6-carboxyfluorescein succinimidylester (CFSE) fluorescent dye $(5 \mu \mathrm{M})$ in RPMI-1640 medium without FBS for 10 min, while unloaded B16 cells were labeled only with CFSE $(0.5 \mu \mathrm{M})$. CFSE labeling was stopped by the addition of an equal volume of cold FBS for $3 \mathrm{~min}$, and then the cells were washed and counted. CFSE ${ }^{\text {high }}$ and CFSE $^{\text {low }}$-labeled cells were mixed together at a 1:1 ratio, which was confirmed by flow cytometry. Different numbers of splenocytes from vaccinated mice were then incubated with the B16 cells (peptide-loaded $5 \times 10^{4}$, unloaded $5 \times 10^{4}$ ) for $6 \mathrm{~h}$ at $37^{\circ} \mathrm{C}$, after which the co-cultures were analyzed on a FACS MoFlo XDP (Beckman Coulter, USA) for assessment of the percentage of CFSE-labeled B16 cells. Specific killing was calculated as follows: $\%$ killing $=[1$ - $($ peptide - loaded cells $/$ unloaded cells from immunized group)/(peptide - loaded cells/ unloaded cells from naive group)] x100.

Tumor immunotherapy in C57BL/6 mice. Tumor-suppressing experiments were carried out in mice using the following two protocols. C57BL/6 mice (8 per group) were injected subcutaneously with $1 \times 10^{5} \mathrm{~B} 16$ viable cells per mouse on day 0 . Thus, the homologous and heterologous prime-boost regimen was carried out by different strategies. Homologous immunity was conducted by immunizing the mice with 9M+DDA/ MPL three times on days 3,10 and 17. While heterologous immunity was conducted by immunizing the mice with AD-9M $\left(1 \times 10^{8} \mathrm{pfu}\right)$ into the tibialis anterior muscles of both legs (50 $\mu 1$ each) per mouse on day 3 and 9M+DDA/MPL on day 10 and 17. Negative control mice were administered $100 \mu 1$ PBS. Tumor diameters were measured in two dimen- sions every 2 days. Tumor volumes $\left(\mathrm{mm}^{3}\right)$ were calculated as follows: $\mathrm{V}=\left(\mathrm{A}^{2} \mathrm{xB}\right) / 2$, where $\mathrm{A}$ is the length of the short axis and $B$ is the length of the long axis. Mice were sacrificed at week 4 after inoculation with B16 cells to remove and weigh the tumors. Moreover, T cellular immune responses were also detected after the mice were sacrificed. Survival of mice was monitored for $\sim 60$ days.

Cell proliferation. Single cell suspensions of splenocytes at $2 \times 10^{6} / \mathrm{ml}$ in PBS were mixed with an equal volume of $20 \mathrm{mM}$ CFSE in PBS (Molecular Probes). Cells were incubated for $10 \mathrm{~min}$ at room temperature in the dark. Labeling was stopped by addition of FBS and cells were washed twice with PBS. CFSE-labeled spleen cells were cultured at $37^{\circ} \mathrm{C}$ for 5 days and then used for flow cytometry analysis.

Cytokine detection by multiplex flow immunoassay. Splenocytes $\left(1 \times 10^{7}\right)$ from the vaccinated tumor-bearing mice were stimulated with the $9 \mathrm{M}$ protein at a concentration of $5 \mu \mathrm{g} / \mathrm{ml}$ for $15 \mathrm{~h}$, and then the cell culture supernatants were collected to measure multiple cytokines. IL-2 and IL-10 were detected by Bio-Plex pro assays. After the Bio-Plex beads were incubated with the cell culture supernatants in a reaction vessel for half an hour at room temperature in the dark, they were washed and a fluorescent reporter antibody was added to the reaction mixture. Following the second incubation $\sim 40 \mathrm{~min}$ and wash cycle, the beads were suspended in buffer and passed through a flow-based detector.

Statistical analyses. All in vivo and in vitro experiments were performed at least 3 times. Data were analyzed using one-way ANOVA. Differences between the groups were assessed for statistical significance using the unpaired T-test. $\mathrm{P}<0.05$ was considered significant, and $\mathrm{P}<0.01$ was considered highly significant. All statistical analyses were performed with Graphpad Prism software. 
A
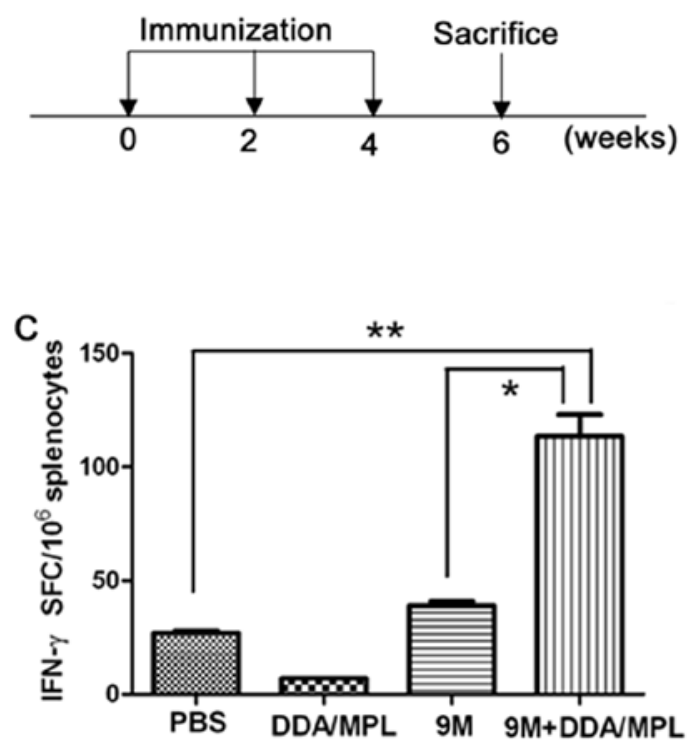

B
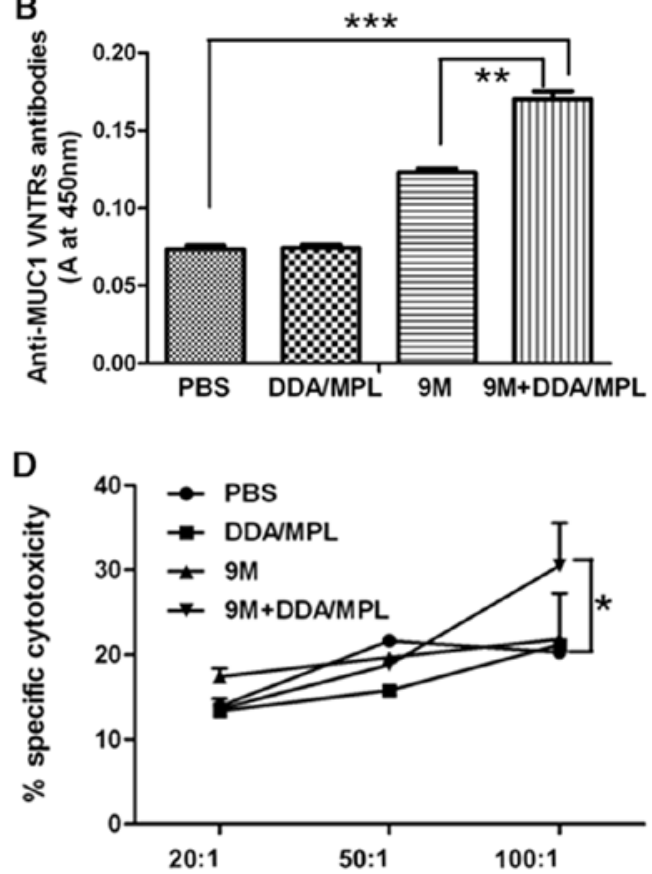

Figure 2. MUC1-specific immunity elicited by vaccination. (A) Immunization schedule. Mice were immunized four times at 2-week intervals. Blood was collected 1 week before the first immunization and 2 weeks after the last immunization when the mice were sacrificed at week 6 . (B) Humoral immunity. Sera diluted 1:100 for testing by ELISA were obtained from each group of C57BL/6 mice vaccinated. (C) Cytolytic activity of splenocytes from immunized mice was determined. (D) IFN- $\gamma$ released by T cells. Splenocytes from vaccinated mice were stimulated with MUC1 protein, and the frequencies of IFN- $\gamma-$ producing $\mathrm{T}$ cells were measured by ELISPOT $\left({ }^{* *} \mathrm{P}<0.01\right)$.

\section{Results}

Construction and expression of $A D-9 M$ and $9 M$ protein. MUC1 VNTRs were also subcloned into the adenovirus vector as previously described (Fig. 1A), and the expression of wild-type adenovirus (wtAD) and recombinant adenovirus expressing MUC1 VNTRs (AD-9M) in HEK293 cells is shown in Fig. 1B. Fig. 1C shows identification of purified MUC1 VNTR protein by western blotting with anti-MUC1 VNTR mAb.

DDA/MPL enhances humoral and cellular immune response induced by the $9 M$ protein vaccine. To investigate the adjuvant effect of DDA/MPL on 9M protein vaccine, groups of mice were immunized with $9 \mathrm{M}+\mathrm{DDA} / \mathrm{MPL}$, the $9 \mathrm{M}$ and DDA/MPL as shown in Fig. 2A. By ELISA, the 9M group showed $\sim 2$-fold higher antibody titer than the PBS and DDA/ MPL group ( $\mathrm{P}>0.05)$; however, serum from the 9M+DDA/ MPL group mice had higher antibody titers than the PBS, DDA/MPL and 9M group $(\mathrm{P}<0.001 ; \mathrm{P}<0.01$, especially), suggesting that DDA/MPL could substantially enhance the humoral immunity induced by protein vaccine (Fig. 2B). Moreover, the trend was also observed in cellular immune response. ELISPOT assay was used to detect the frequency of antigen-specific IFN- $\gamma$-secreting T cells. The results showed that IFN- $\gamma$ induced by the $9 \mathrm{M}$ protein group was no more than the PBS group $(\mathrm{P}>0.05)$; however, the response in mice vaccinated with recombinant $9 \mathrm{M}$ protein and DDA/MPL was significantly higher (nearly three times) than that observed for mice given separately the $9 \mathrm{M}$ protein and DDA/MPL $(\mathrm{P}<0.05)$ (Fig. 2C). Specific killing was measured after incubating the

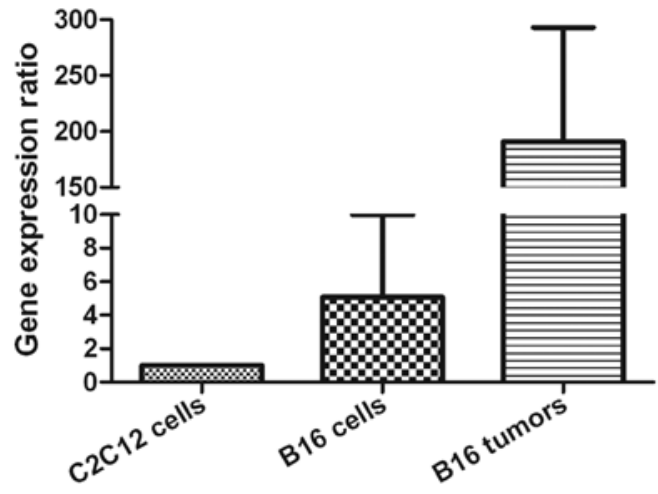

Figure 3. MUC1 expression in B16 cells and B16 tissues. Comparison of gene expression ratio in $\mathrm{C} 2 \mathrm{C} 12$ cells, B16 cells and B16 tumors using qRT-PCR using $\beta$-actin as endogenous control.

splenocytes for $2 \mathrm{~h}$ with SAPDTRPAP-pulsed CFSE-labeled B16 cells (target cells). The mixture of 9M and DDA/MPL induced higher specific cytotoxic $\mathrm{CD} 8^{+} \mathrm{T}$ cell (CTL) response in mice than separately used when the ratio of effector cells to target cells was 100:1 (Fig. 2D). Therefore, DDA/MPL served as an effective adjuvant for 9M protein vaccine for enhanced humoral and cellular immune response. However, DDA/ MPL did not enhance tumor growth inhibition and extend the survival of melanoma-bearing mice.

The expression level of MUC1 was evaluated in B16 cells as well as $\mathrm{C} 2 \mathrm{C} 12$ cells. The results indicated that $\mathrm{B} 16$ cells and implanted B16 tumors showed nearly 5 -fold and 200 -fold higher mRNA levels compared to $\mathrm{C} 2 \mathrm{C} 12$ cells separately (Fig. 3), 

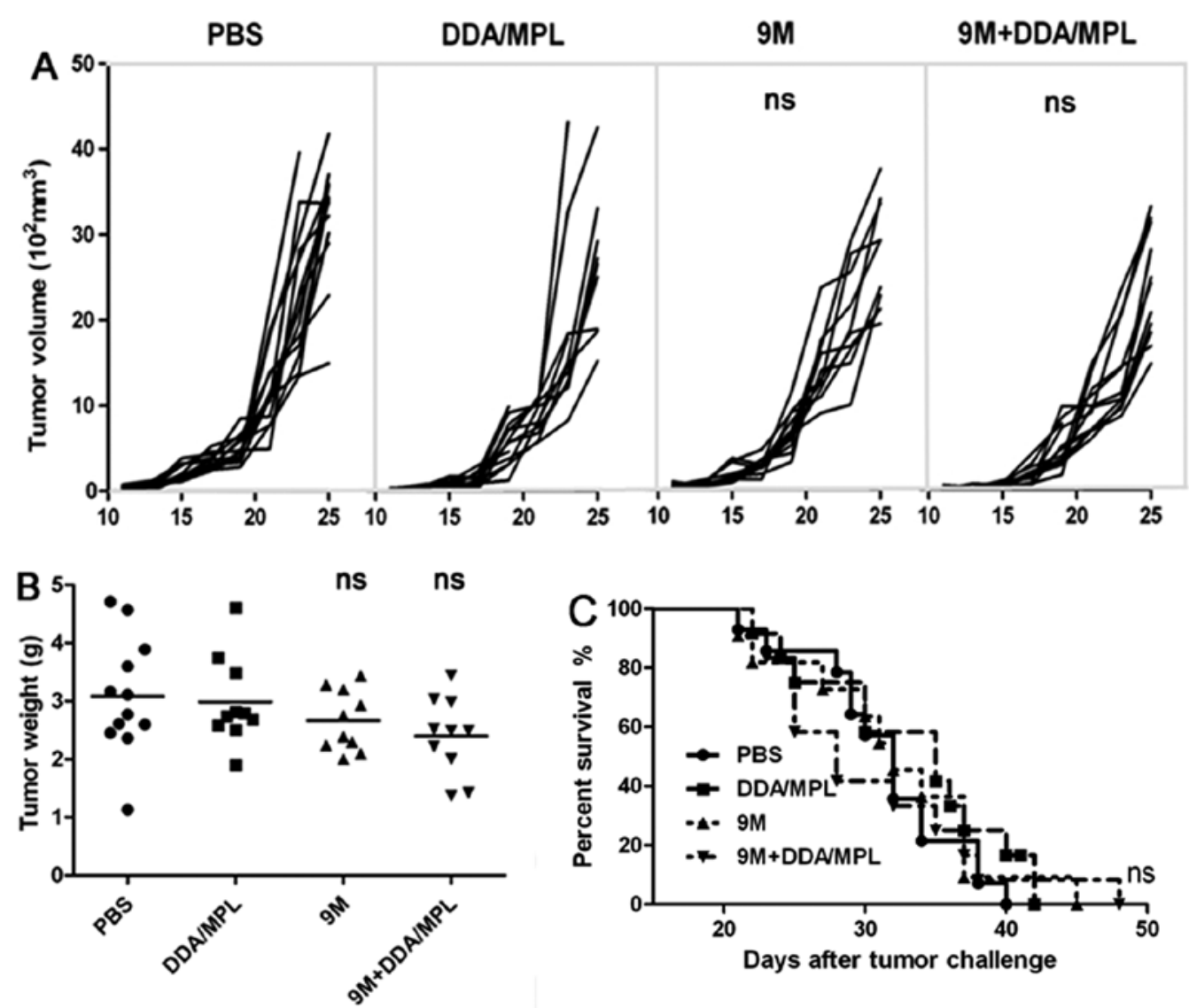

Figure 4. Therapeutic antitumor effects induced by AD-9M/9M+DDA/MPL in subcutaneous B16 melanoma-bearing mice. (A) Tumor growth curves. Each line represents tumor growth kinetics in each mouse. (B) On the 26th day after tumor challenge, mice were sacrificed to measure tumor weights. Tumor weights denoted as means \pm SD were: PBS group, $5.87 \pm 1.66 \mathrm{~g}$; AD-9M group, $2.24 \pm 2.27 \mathrm{~g} ;$ AD-9M/9M+DDA/MPL group, $1.18 \pm 0.78 \mathrm{~g}\left({ }^{* *} \mathrm{P}<0.01 ;{ }^{* * * *} \mathrm{P}<0.001\right)$. (C) Survival curves. Survival of the mice was calculated $\left.{ }^{* *} \mathrm{P}<0.01 ;{ }^{* * * *} \mathrm{P}<0.001\right)$. The mean survival times were: PBS group, 27 days; AD-9M group, 33 days; AD-9M/9M+DDA/MPL group, 37 days $(\mathrm{P}<0.05)$.

indicating that MUC1 was overexpressed in melanoma tissues and cells than normal myoblast cells. To evaluate whether the 9M+DDA/MPL vaccine had inhibitory effects on tumor growth, C57BL/6 mice (10 per group) were inoculated with B16 cells $\left(1 \times 10^{5}\right)$ on day 0 . After tumor establishment, the mice were immunized with PBS, 9M, DDA/MPL, 9M+DDA/MPL on days 3,10 and 17. Tumor growth was monitored for 28 days after inoculation. As shown in Fig. 4A-C, 9M+DDA/MPL homologous immunization strategy did not inhibit tumor growth and extend the survival of melanoma-bearing mice, and the difference was not significant $(\mathrm{P}>0.05)$.

VPP vaccine displays anti-melanoma effect in mice. As the adjuvant vaccine did not significantly enhance the antitumor activity, immunization strategy was studied. Our previous data showed that recombinant adenovirus prime protein boost heterologous immunization strategy showed superior antitumor effects than homologous immunization (11). Thus, an adenoviral prime protein boost immunization (VPP) was conducted in this study. Mice were inoculated with $1 \times 10^{5} \mathrm{~B} 16$ cells at day 0 into the right hind leg. After the tumor was established, the mice were immunized with $\mathrm{AD}-9 \mathrm{M}$ at day 3, and $9 \mathrm{M}+\mathrm{DDA} / \mathrm{MPL}$ at day 10 and 17 . Tumor volume in the subcutaneous B16 melanoma-bearing mice was measured every other day and the survival of each treated mouse was monitored.
As shown in Fig. 5A, both AD-9M and AD-9M/9M+DDA/ MPL VPP vaccine induced growth inhibition of the tumor $(\mathrm{P}<0.01 ; \mathrm{P}<0.001)$ compared with PBS. According to tumor weight (Fig. 5B), the AD-9M/9M+DDA/MPL strategy showed a tumor inhibition ratio of $59.45 \%$ to the PBS group $(\mathrm{P}<0.05)$. The AD-9M group showed a tumor inhibition ratio of $39.86 \%$ to the PBS group. In parallel, a significant prolonged survival was recorded in the mice immunized with AD-9M/9M+DDA/MPL VPP vaccines. As shown in Fig. 5C, by day 36 and 38 after B16 melanoma inoculation, all mice in the PBS and AD-9M groups died. Until day 50 after tumor challenge, $30 \%$ of the mice immunized with AD-9M/9M+DDA/MPL VPP vaccines survived. The survival rate of melanoma-bearing mice vaccinated with AD-9M/9M+DDA/MPL was 33.33\% $(\mathrm{P}<0.05)$, whereas the survival rate of mice vaccinated with $\mathrm{AD}-9 \mathrm{M}$ was $22.22 \%(\mathrm{P}>0.05)$.

Antitumor mechanism of the vaccine. To investigate the antitumor mechanism of the vaccine, lymphocyte proliferation and cytokines were detected. It was shown that the ability of promoting lymphocyte proliferation of the AD-9M/9M+DDA/MPL group was >3-fold compared to the PBS group, while that of the AD-9M group was nearly 2-fold compared to PBS (Fig. 6A). IL-2 and IL-10 concentration $(\mathrm{pg} / \mathrm{ml})$ in the culture supernatant after stimulation with $9 \mathrm{M}$ 
A
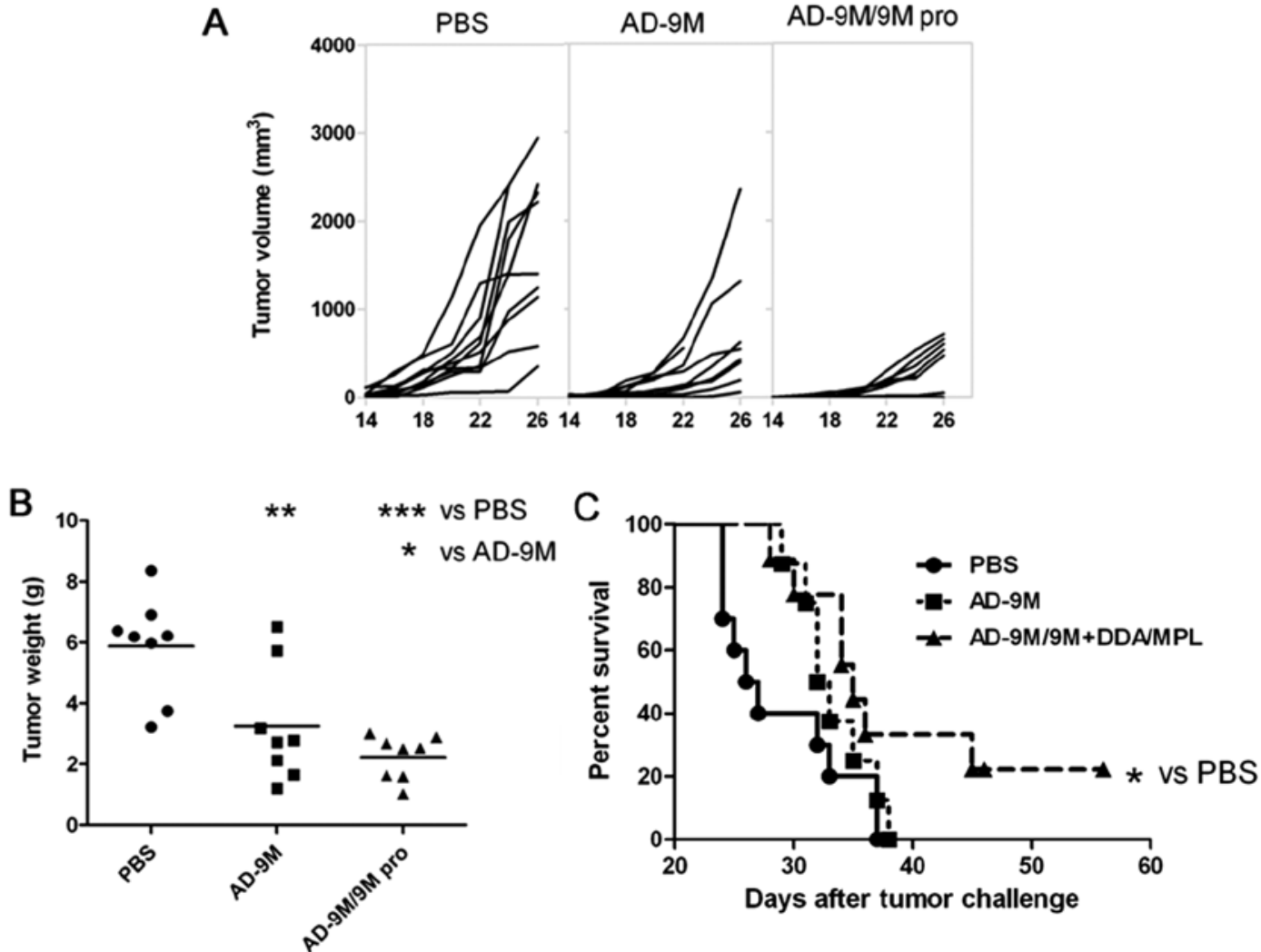

Figure 5. Antitumor effects of vaccination with the $9 \mathrm{M}$ protein combined with DDA/MPL as an adjuvant. (A) Tumor volumes were measured every other day until day 27. (B) On the 26th day after tumor challenge, mice were sacrificed to measure tumor weights. Tumor weights denoted as mean \pm SD were: PBS group, $3.08 \pm 1.00 \mathrm{~g}$; DDA/MPL group, $2.99 \pm 0.76 \mathrm{~g}$; $9 \mathrm{M}$ group, $2.66 \pm 0.53 \mathrm{~g}$; and 9M+DDA/MPL group, $2.40 \pm 0.67 \mathrm{~g}$. (C) Survival was monitored for 50 days. The mean survival times were: PBS group, 32 days; DDA/MPL group, 34 days; $9 \mathrm{M}$ group, 32 days; $9 \mathrm{M}+\mathrm{DDA} / \mathrm{MPL}$ group, 33 days.
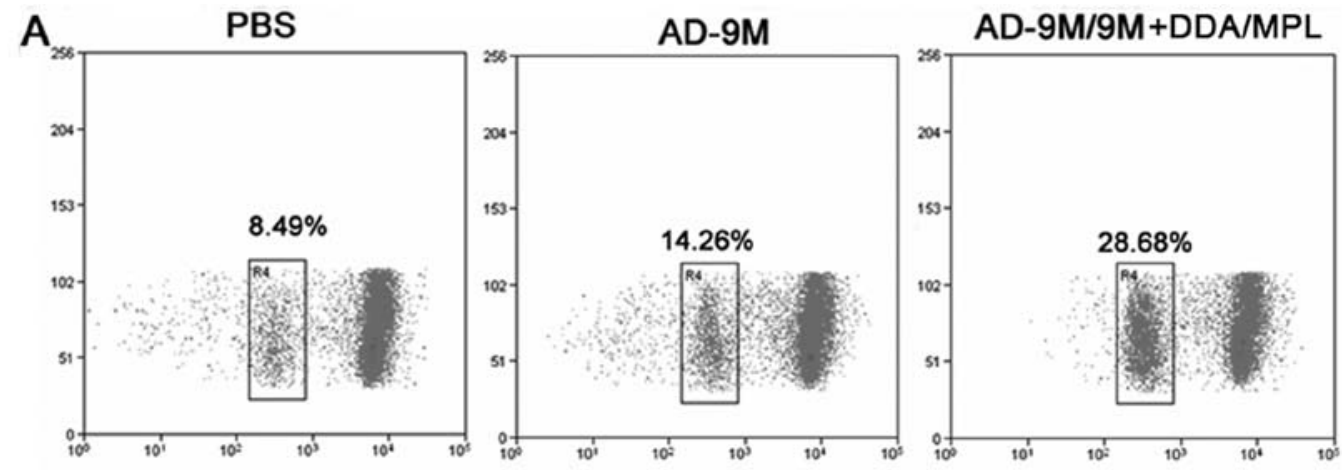

B
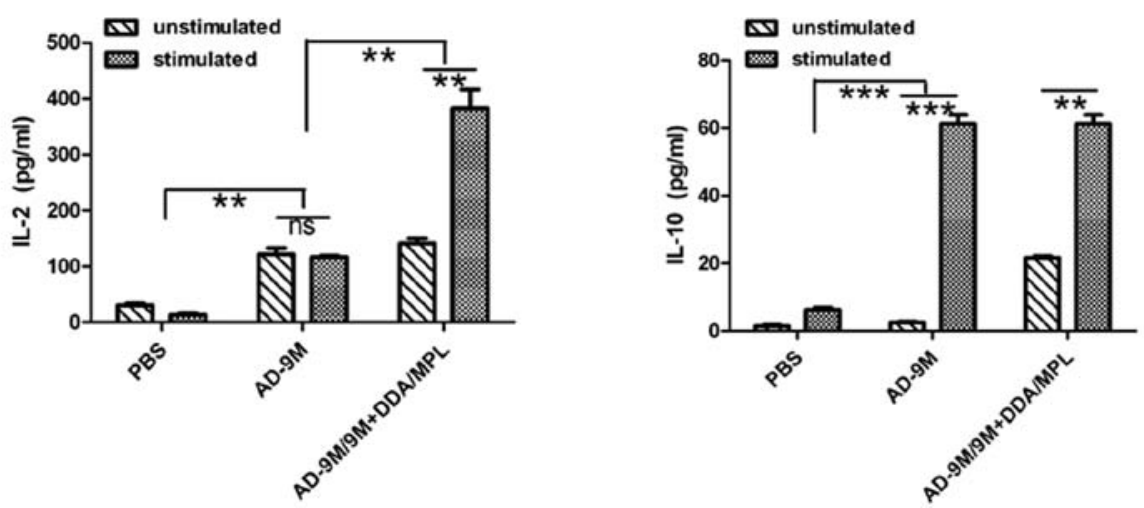

Figure 6. Antitumor mechanism of the vaccine. (A) After isolation, splenocytes were labeled with CFSE at a concentration of $10 \mu \mathrm{M}$. Cell proliferation was evaluated by flow cytometry after incubation for 5 days. (B) IL-2 and IL-10 were measured using BioPlex ${ }^{\mathrm{TM}} 2200$. Vaccination with AD-9M/9M+DDA/MPL elicited significant levels of antigen-specific splenocytes secreting both IL-2 and IL-10 cytokines. Unstimulated indicates splenocytes incubated with RPMI-1640; stimulated indicates splenocytes incubated with survivin protein. 
protein were detected simultaneously using the BioPlex ${ }^{\mathrm{TM}}$ 2200 (Fig. 6B). Both the AD-9M and AD-9M/9M+DDA/ MPL groups elicited $~ 6$-fold higher IL-10 level than the PBS group $(\mathrm{P}<0.01)$, and there was no difference between the two groups $(\mathrm{P}>0.05)$. However, AD-9M enhanced $>3$-fold IL-2 levels than the PBS group $(\mathrm{P}<0.01)$, while the VPP vaccine enhanced $\sim 13$-fold to the PBS group ( $\mathrm{P}<0.001)$ (Fig. 6B). The VPP vaccine group showed $\sim 2.5$-fold IL-2 concentration to the AD-9M group $(\mathrm{P}<0.001$ and $\mathrm{P}<0.01$, especially) and the AD-9M vaccine group showed $\sim 3$-fold IL-2 concentration to PBS. IL-10 was a Th2-type cytokine and IL-2 was a Th1-type cytokine. It was suggested that the AD-9M and the VPP vaccine in the tumor-challenged mice may enhance both Th1 and Th2 type immune response. However, the VPP vaccine enhanced more Th1 type immune response than Th2 type immune response, which may be more beneficial to antitumor effects.

\section{Discussion}

In the present study, we sought to prepare a recombinant adenovirus vector expressing MUC1 and to select a proper adjuvant (DDA/MPL) to the recombinant MUC1 protein vaccine. Adenoviral prime protein boost immunization induced an efficient antitumor effect in a murine melanoma model. Moreover, this antitumor effect might be predominantly mediated by Th1-type immune response. The data suggested that adenoviral prime protein plus adjuvant boost immunization strategy may be a potential treatment against melanoma.

MPL formulated with DDA liposomes (DDA/MPL) is not approved for clinical trials as an adjuvant, it has been shown to induce a significant level of protective immunity in Chlamydia and tuberculosis vaccine research $(17,18)$. In this study, DDA/MPL was selected as an adjuvant for the MUC1 VNTR-based protein vaccine to evaluate its ability to enhance immune response in our study. The data showed that DDA/ MPL could effectively enhance the cellular and humoral immunity against MUC1 protein.

Adenovirus (AD) has been clinically evaluated and proven to be immunogenic and safe (19). As a classic tumor-associated antigen, MUC1 has been studied for many years both in preclinical and clinical trials. MUC1 has been prioritized as a cancer vaccine target antigen (20). In our study, a recombinant adenovirus expressing MUC1 was constructed and evaluated for the anti-melanoma effect. The results showed that less tumor inhibition was induced by the AD-9M than the VPP vaccine, which might be caused by different Th types.

It is well accepted that multiple immunizations (i.e. 'primeboost') are crucial for even the most successful vaccines. In many cases, heterologous prime-boost can be more immunogenic than homologous prime-boost (21). Our previous data also showed that heterologous adenovirus prime-protein boost strategy displayed more suppressive effects of tumor growth. In this study, homologous immunization strategy could not inhibit tumor growth and extend the survival of melanomabearing mice (Fig. 4A-C). However, AD-9M prime-9M+DDA/ MPL boost immunization was conducted for effect on tumor volume and survival of challenged C57BL/6 mice. The results showed the VPP vaccine could inhibit tumor growth of melanoma model mice in the tumor volume and weight inhibition
(Fig. 5A and B). Moreover, the VPP vaccine could prolong survival time effectively (Fig. 5C). The survival time of VPP vaccine increased $33.33 \%$ to $\mathrm{PBS}$ group $(\mathrm{P}<0.05)$, yet $\mathrm{AD}-9 \mathrm{M}$ increased $22.22 \%(\mathrm{P}>0.05)$.

To investigate the mechanism of antitumor effects, splenocyte proliferation and elicited cytokines were evaluated. The AD-9M/9M+DDA/MPL VPP vaccine group showed 3-fold and 2-fold proliferation rates to $\mathrm{PBS}$ and $\mathrm{AD}-9 \mathrm{M}$ groups (Fig. 6A). The concentration of IL-10 after stimulation was $60.5 \mathrm{pg} / \mathrm{ml}$, which was similar to the AD-9M group. The IL-2 concentration of the AD-9M/9M+DDA/MPL VPP vaccine group after inoculation with $9 \mathrm{M}$ protein was $382 \mathrm{pg} / \mathrm{ml}$, which was 2.5-fold higher than the AD-9M groups (Fig. 6B). Moreover, IL-2 was a Th1 type cytokine and IL-10 was a Th2 type cytokine. We speculated that both Th1 and Th2 type immune response play an important role in tumor inhibition, especially the IL-2 biased Th1 type.

In summary, DDA/MPL could significantly enhance the cellular and humoral immunity based on 9M protein. However, the homologous protein prime-boost strategy applied vaccine (PPP) could slightly inhibit the tumor growth and prolong the survival of tumor-bearing mice, but the difference was not significant $(\mathrm{P}>0.05)$. However, adenoviral vector primerecombinant protein and DDA/MPL boost immune strategy (VPP) exerted significant anti-melanoma effects in mice. It was shown that the MUC1 VPP vaccine was able to inhibit the growth of B16 melanoma in mice and to prolong the survival of B16 melanoma-bearing mice $(\mathrm{P}<0.05)$. The data suggested that the VPP strategy combined with DDA/MPL adjuvant might be a simple and efficient way to produce efficient MUC1-based vaccines for melanomas or other tumors.

\section{Acknowledgements}

This study was supported by the Specialized Research Fund for the National Natural Science Foundation of China (no. 31300765), the Specialized Research Fund for the Doctoral Program of Higher Education (New Teachers) (grant no. 20120061120025), Jilin Province Science and Technology Development Program (no. 20130522006JH), and the National Science and Technology Major Project of the Ministry of Science and Technology of China (no. 2012ZX10001009-002).

\section{References}

1. Tang CK and Apostolopoulos V: Strategies used for MUC1 immunotherapy: preclinical studies. Expert Rev Vaccines 7: 951-962, 2008.

2. Tang CK, Katsara M and Apostolopoulos V: Strategies used for MUC1 immunotherapy: human clinical studies. Expert Rev Vaccines 7: 963-975, 2008.

3. Snyder LA, Goletz TJ, Gunn GR, et al: A MUC1/IL-18 DNA vaccine induces anti-tumor immunity and increased survival in MUC1 transgenic mice. Vaccine 24: 3340-3352, 2006.

4. Tarp MA, Sorensen AL, Mandel U, et al: Identification of a novel cancer-specific immunodominant glycopeptide epitope in the MUC1 tandem repeat. Glycobiology 17: 197-209, 2007.

5. Yuan S, Shi C, Lv Y, Wang T, Wang $\mathrm{H}$ and Han W: A novel Bacillus Calmette-Guérin-based breast cancer vaccine that coexpresses multiple tandem repeats of MUC1 and CD80 breaks the immune tolerance and inhibits MUC1-positive breast cancer growth. Cancer Biother Radiopharm 24: 607-613, 2009.

6. Mukherjee P, Basu GD, Tinder TL, et al: Progression of pancreatic adenocarcinoma is significantly impeded with a combination of vaccine and COX-2 inhibition. J Immunol 182: 216-224, 2009. 
7. Nicholls EF, Madera L and Hancock RE: Immunomodulators as adjuvants for vaccines and antimicrobial therapy. Ann NY Acad Sci 1213: 46-61, 2010.

8. Korsholm KS, Agger EM, Foged C, et al: The adjuvant mechanism of cationic dimethyldioctadecylammonium liposomes. Immunology 121: 216-226, 2007.

9. Persing DH, Coler RN, Lacy MJ, et al: Taking toll: lipid A mimetics as adjuvants and immunomodulators. Trends Microbiol 10: S32-S37, 2002.

10. Takeuchi O, Hoshino K, Kawai T, et al: Differential roles of TLR2 and TLR4 in recognition of gram-negative and grampositive bacterial cell wall components. Immunity 11: 443-451, 1999.

11. Wang YQ, Zhang HH, Liu CL, et al: Enhancement of survivinspecific anti-tumor immunity by adenovirus prime protein-boost immunity strategy with DDA/MPL adjuvant in a murine melanoma model. Int Immunopharmacol 17: 9-17, 2013.

12. Harari A, Bart PA, Stohr W, et al: An HIV-1 clade C DNA prime, NYVAC boost vaccine regimen induces reliable, polyfunctional, and long-lasting T cell responses. J Exp Med 205: 63-77, 2008.

13. Magalhaes I, Sizemore DR, Ahmed RK, et al: rBCG induces strong antigen-specific $\mathrm{T}$ cell responses in rhesus macaques in a prime-boost setting with an adenovirus 35 tuberculosis vaccine vector. PLoS One 3: e3790, 2008.

14. Garcia-Hernandez Mde L, Gray A, Hubby B and Kast WM In vivo effects of vaccination with six-transmembrane epithelial antigen of the prostate: a candidate antigen for treating prostate cancer. Cancer Res 67: 1344-1351, 2007.
15. Yu B, Zhang Y, Zhan Y, et al: Co-expression of herpes simplex virus thymidine kinase and Escherichia coli nitroreductase by an hTERT-driven adenovirus vector in breast cancer cells results in additive anti-tumor effects. Oncol Rep 26: 255-264, 2011.

16. You Q, Jiang C, Wu Y, et al: Subcutaneous administration of modified vaccinia virus Ankara expressing an Ag85B-ESAT6 fusion protein, but not an adenovirus-based vaccine, protects mice against intravenous challenge with Mycobacterium tuberculosis. Scand J Immunol 75: 77-84, 2012.

17. Yu H, Karunakaran KP, Jiang X, Shen C, Andersen $P$ and Brunham RC: Chlamydia muridarum $\mathrm{T}$ cell antigens and adjuvants that induce protective immunity in mice. Infect Immun 80: $1510-1518,2012$

18. Kolibab K, Parra M, Yang AL, Perera LP, Derrick SC and Morris SL: A practical in vitro growth inhibition assay for the evaluation of TB vaccines. Vaccine 28: 317-322, 2009.

19. de Gruijl TD and van de Ven R: Chapter six - Adenovirus-based immunotherapy of cancer: promises to keep. Adv Cancer Res 115: 147-220, 2012.

20. Cheever MA, Allison JP, Ferris AS, et al: The prioritization of cancer antigens: a national cancer institute pilot project for the acceleration of translational research. Clin Cancer Res 15: 5323-5337, 2009.

21. Lu S: Heterologous prime-boost vaccination. Curr Opin Immunol 21: 346-351, 2009. 